\title{
Properties of Water at High Pressures and Low Temperatures- $\mathbf{I}^{*}$
}

\author{
Five Different Kinds of Ice Discovered
}

\section{By P. W. Bridgman, Ph.D., Jefferson Physical Laboratory, Harvard University}

The experiments which I am about to describe are experiments in the field of very high pressures, which is a practically new field. Under conditions of high pressure, many of the ordinary properties of matter are changed; and the bursting strength of vessels in which such pressures are produced is found to bear no relation to their strength under ordinary conditions. In conducting the following investigations on the effect of very high pressures on water, it was found necessary to make

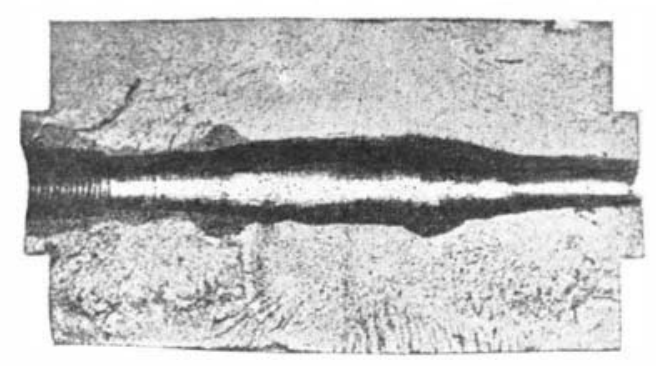

Fig. 1.-One of the halves of a cylinder of tool steel split by the application of internal pressure. The inner hole has stretched from one half inch to one and one fifth inches. The maximum pressure one and one fifth inches. The maximum pressure
withstood by this cylinder was 30,000 atmospheres.

many preliminary experiments on the strength of the containing vessels before accurate measurements of pressure could be made. In the course of this preliminary work many interesting facts concerning the behavio of materials under high pressures were disclosed. In this paper will be given, first, some of the results of the preliminary experiments on the strength of the containers and then a description of the experiments made to ascertain the effect of high pressures on water. The paper will be, in large part, a record of my own experimental work.

By way of introduction, it is perhaps desirable to give some idea of the magnitude of the pressures involved. The highest pressures which are ordinarily familiar to us are probably those of modern high-power artillery; the average firing pressure exerted in many of our large guns is about 2,000 atmospheres, or 30,000 pounds per square inch. The highest pressures reached in the experiments which I am about to describe are ten times this amount; that is, 20,000 atmospheres, or 300,000 pounds per square inch. A pressure of 20,000 atmospheres, if exerted against a rod of steel one inch square, would enable it to support a locomotive weighing 150 tons. The highest pressure I know of that has been previously measured is 10,000 atmospheres; this was proviously measured is 10,000 atmospheres; this was vessel; it is about one half the pressure recorded in the present experiments. Nitroglycerin subjected to the pressures attained in the following experiments would lose all ability to explode. The pressure exerted at the bottom of the ocean at, say, a depth of six miles, is about 1,000 atmospheres; a pressure of 20,000 atmospheres would, therefore, be exerted at the bottom of an ocean would, therefore, be exerted at the bottom of an ocean
120 miles deep. If the average density of the rocks of the earth's crust is taken as 2.5, 20,000 atmospheres is the pressure which prevails at a depth of fifty miles below the surface of the earth.

It should be borne in mind that in all the experiments made, the pressures were produced in a liquid, which must be held in a container. It is a comparatively simple must be held in a container. It is a comparatively simple
matter to produce pressures as high as 300,000 pounds matter to produce pressures as high as 300,000 pound
per square inch in a solid piece of steel, but it is another matter to maintain such a pressure in a liquid and pre vent all leaking of the latter from the container.

The most essential part of the preliminary work was to design a packing that would keep the vessel in which the pressures were to be produced absolutely tight, and prevent the liquid from leaking from it. The feature of the form of packing finally designed is that it is mad tighter and tighter by the pressure in the vessel itself the greater the pressure obtaining in the vessel, the less can the liquid leak. Most of the packing in ordinary use is made tight by compressing it by means of a screw a packing so compressed will leak as soon as a pressur is exterted on the liquid it holds back equal to that initially produced on it by the screw; further, the presinitially produced on it by the screw; further, the pres-
sures that are here dealt with are much greater than any sures that are here dealt with are much greater than any
pressures it is possible to produce by such means. The ordinary screw-tightened packing is, therefore, always certain to leak. With a packing that tightens itself by *Abstract of paper presented at the meeting of the Section of
Physics and Chemistry, and publshed in the Journal of the Eranklin Institute. the pressure in the vessel, however, the only limit to the pressures that can be reached is set by the strength of the vessel itself.

The second part of the preliminary work consisted in finding what limits of pressure a steel vessel will support steel being selected as the best material of which to mak the container. In all the experiments the pressures were produced by pushing a piston, by means of a hydraulic press, into the cylinder containing the liquid, and this means of producing the pressure immediately introduces two essentially different problems. The first is the consideration of the strength of the steel piston, and the second that of the strength of the steel cylinder. These problems are independent, as these two parts of the apparatus are subjected to different strain. In the piston the strain is one of compression, while in the cylinder it is one of bursting, or tension.

It was found by experiment that the best material for the piston was glass-hard steel. A steel that is left glass-hard is usually too brittle to use, and its temper has to be drawn, but this process makes the steel a little softer, so that it will not support so high a compression as when left glass-hard. Under the conditions in the experiments the piston was subjected to a direct compression, without any tendency to bend, so that its brittleness, which would be detrimental under conditions of compound stress, was not here objectionable. Steel from many different manufacturers was tried, and the best grades of steel for this purpose were found to be steels which contained some chromium as well as high

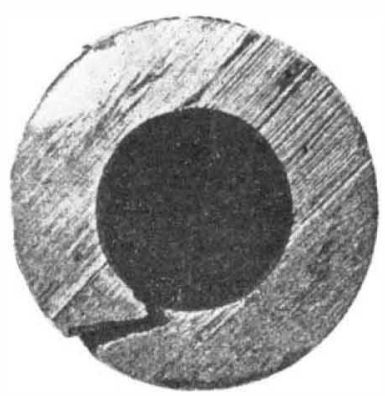

Fig. 2.-Cross section of a cylinder of Bessemer steel ruptured by the application of internal pressure. This cylinder was originally two inches outside and one half inch inside diameter. The inner-hole has been stretched to one and three inner hole has
eighth inches.

carbon. The compression that a piece of glass-hard steel will support when it is held rigidly so that it will not bend is surprisingly large; several grades of steel were found that would support a compression of 600,000 pounds per square inch, and one grade supported as high as 750,000 pounds per square inch. It will be noticed that these pressures are about twice as great as the highest pressures recorded in the present experiments. The problem of the strength of the piston did not, tberefore, prove of difficulty.

The strength of the steel cylinders was also a factor which had to be settled by experiment, since it was found that no theory of the strength of a cylinder is of any value for very high pressures. All ordinary theories predict that no cylinder can be stressed to more than the tensile strength of the steel, no matter how thick are its walls; thus if the tensile strength of the steel is 150,000 pounds per square inch (as it is for many grades of high carbon steel), then theory predicts that it is impossible to maintain a pressure of more than 150,000 pounds per square inch in a cylinder, even with infinitely thick walls. It is, however, of no particular use to make a cylinder of more than a certain thickness, for the reason that the inside layers support most of the pressure; the outside layers of the material of a very thick cylinder do not take up the stress of the inner layers, and the cylinder flows at the inside at a certain pressure. A fow rough experiments showed the actual pressure that a cylinder can support to be much in excess of that predicted by the ordinary theory; this is on account of the fact that when the pressure reaches a certain value the inner layers do not break, but stretch, and thus allow the outer layers to assume some of their share of the load. It was found that the most efficient way to make a cylinder support a high pressure was first to stretch it on the inside by applying a much higher pressure than it was intended to maintain in practice, and then to machine it to its final diameter. A cylinder treated in this way is in a state of internal strain, exactly as is a gun which has hoops shrunk on it from the outside, the tension in the hoops inducing initial compression in the interior of the gun. When pressure is produced in such a gun, it removes the compression from the inner layers of the material and the tension of the outer layers is increased. But it has been shown that the tension in the inner layers increases more rapidly than that in the outer, and it can be seen, therefore, that in time, the increasing tension in the inner layers neutralizes the compression which existed there initially and eventually equals the

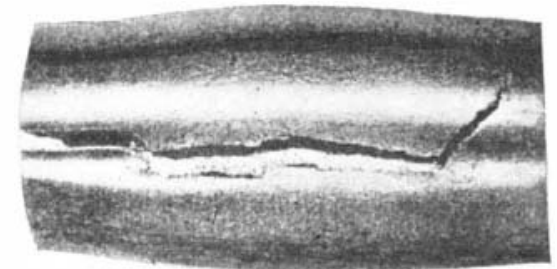

Fig. 3.-View of the outside of the cylinder shown

in Fig. 2, taken before the section was made.

increasing tension of the outer layers, with the result that finally the stress throughout the mass of the cylinder is one of uniform tension. In an ideal condition all parts of the cylinder would be ready to break at the same time, and then the maximum possible strength would result; in any actual case it is, of course, impossible to reach this ideal, but, with the cylinders subjected to a preliminary stretching, it can be approached much more nearly than even in a built-up gun. There are other considerations, which need not be gone into here, which come into force when the cylinder becomes very thick. In actual practice, as already stated, very little is gained in making the cylinder of more than a certain thickness

The best steel to use for the cylinder is found to be a steel-which will stretch considerably before breaking, but which has, at the same time, a high tensile strength. The glass-hard steel used for the piston would not serve at all for this purpose, as a cylinder made of such material would inevitably stretching, and so would not allow the outer layers to assume their share of the load. The steels found most suitable are those tough steels that have been put upon the market to meet the demands of the manufacturers of automobiles. One steel made by Krupp was found very good, but best of all was a steel made in this country by the electric furnace method; this steel is a chrome vanadium steel, and has, when hardened in oil, a tensile strength that may reach as high as 300,000 pounds per square inch. The highest pressure that I have ever found it possible to reach in a cylinder has been 40,000 atmospheres, or twice the highest pressures at which I have made accurate measurements.

In the preliminary work on steel cylinders, many cylinders were broken. This gave opportunity for interesting observations on the manner of rupture at high pressures, and two facts not to be expected according to ordinary theories were noted. The first was the enormous amount of stretch that the steel at the inner layer of a cylinder will support without rupture; this is well shown in Figs. 1, 2, and 3. In the first figure the cylinder was originally one half inch in diameter, but it stretched to one and one fifth inches before breaking. This same specimen of steel, if tested for tensile strength in the ordinary way, would have broken when the strain had become about 30 per cent, whereas here the strain was about 140 per cent before the rupture took place. The second observation was that in all the cylinders tested the break started at the outside, where the stress and the strain are both least; this was observed in all the steels used. There is reason to believe, however, that very brittle substances like glass would break at the inside, as predicted by the ordinary theory. The fact seems to be that if the substance is brittle it will break at the inside first, but that if it is at all plastic it will break at the outside first, the crack traveling inward: In addition to the data obtained regarding the manner in which materials break at high pressures, many other peculiar facts were noted during these preliminary tests. Perhaps the most interesting of these is the increase in rigidity experienced in substances ordinarily soft and pliable. A striking example of this is afforded in the case of paraffin, which under pressures as high as 20,000 atmospheres becomes more rigid than soft steel, so that if paraffin is forced to flow by the application of a very high pressure, and a piece of soft steel is imbedded in it, the steel will flow with the paraffin and will become distorted and twisted with the latter. Soft rubber also becomes very hard and brittle under high pressure; in one experiment a soft rubber washer became so brittle 
that it cracked like glass, and a soft steel washer in contact with the rubber was forced by the pressure in ridges into the cracks in the rubber, thus showing that the rubber had become harder than the steel.

At the beginning of the experiments it was feared that the steel of the cylinder might become porous under very high pressure, causing the liquid to be forced through the solid walls. No instances were found, however, in which the liquid had been forced, by pressure, into the solid metal to any appreciable depth. Many instances have occurred in which a liquid has been blown in a solid stream through apparently solid steel, but all such cases have been traced to flaws in the steel. The only apparent exception is the impossibility of retaining mercury in a cylinder under a high pressure, as the mercury invariably finds its way through the walls and usually ruptures the eylinder. The explanation of this was found to be, however, that the mercury amalgamates with the steel under high pressure, so that this is not properly a case of porosity.

Another all-important task in the preliminary experi- ments, in addition to that of finding what pressures the steel vessels could stand, was to devise some way of accurately measuring the pressure. It need hardly be said that none of the ordinary pressure gages are of the slightest service at these high pressures, and some means of measuring pressures other than with an ordinary gage had, therefore, to be devised. The very simplest method that can be conceived proved to be the best in this case. It consists in inserting a steel piston through a hole in the wall of the cylinder and measuring the force necessary to prevent it from being blown out by the pressure within. There are many mechanical difficulties in realizing such a method as this, the most obvious being to overcom leakage. To do this the piston must fit the hole tightly, but at the same time must fit so freely that there is not enough friction to destroy the accuracy of the reading obtained by its means. It was found possible, by using a small-diameter piston fitting into a comparatively long hole, to take care of both these factors. With th gage as finally constructed, pressures up to 13,000 kilogrammes per square centimeter were measured with an accuracy of one tenth per cent. After high pressures had been successfully measured with such a gage, it was found possible to construct gages of a much more convenient form for actual use, and to calibrate them against this, which became an "absolute" gage. One gage that I have used in most of my later work is a manganin resistance gage, which consists of a coil of manganin wire placed in the pressure eylinder and connected through insulated leads with apparatus for measuring the resistance. The electrical resistance of this coil is found to change with changes of pressure in the eylinder. In increases as the pressure increases, and by comparing with the absolute gage the increase was found to be almost exactly proportional to the increase of pressure. On account of this, manganin is a very much more convenient material to use than any pure metal, since the resistance of all pure metals decreases as the pressure increases, and the decrease is, moreover, not proportional to the increase of pressure.

(To be continued.)

\section{How a Carbon Dioxide Recorder Works*} By Charles H. Bromley

When you use a sponge to soak up water from the floor you do not consider that anything remarkable has happened. You have seen the tanks at the gas works rise as they filled with gas, and drop down as the gas was discharged, and you thought that a usual proceeding.

The principal reasons why a $\mathrm{CO}_{2}$ recorder records $\mathrm{CO}$ are really no more mysterious nor remarkable than the absorption of water by a sponge, or the inflation of a tank by gas. Practically, the same physical actions occur in by gas. Practically, the same physical actions
both cases, but the things we use are different.

Because chemicals have unfamiliar names, is no reason why we should shy at them any more than we shy at a yeast cake, which is a chemical. Some chemical solution absorb certain gases just as a sponge absorbs water. You know that to relieve a gas-bound ammonia pump you let the gas discharge into a pail of water. If the gas were discharged into the noom the fumes would bo quite disagreeable, but when discharged into the water the gas is absorbed like water by a sponge.

Platinum sponge will absorb a surprisingly large amount of hydrogen gas, and the sponge will not increas in volume. To prove that it will absorb this gas we will arrange two bottles, as in Fig. 1. One is filled with water, the other with hydrogen gas. We suspend a piece of platinum sponge in the gas as shown. The sponge will platinum sponge in the gas as shown. The sponge will then absorb the gas, create a vacuum in

draw into it water from the other bottle.
The first experiment showed that some substances do absorb gas. We will now experiment with a sample of flue gas of 100 cubic centimeters; 12 per cent is $\mathrm{CO}$ and the other 88 per cent is made up of other gases.

We have four bottles, Fig. 2, one containing 100 cubic centimeters of water; one, 100 cubic centimeters of flue gas; one with a caustic-potash solution, and the last bottle we will consider inverted and full of water. Opening the pinch cocks on the rubber tubes connecting th bottles, lift the water bottle to the position indicated by the dotted lines. The water flows into the second bottle, forcing the 100 cubic centimeters of flue gas out causing it to bubble up through the caustic-potash solution. After the gas has passed through the solution, an analysis would show no $\mathrm{CO}_{2}$, but it would show that 88 cubic centimeters of other gases were present At the beginning of the experiment the inverted bottlo is filled with water and is sealed by a little water in the jar, just as the spring-water-bottle drinking fountains are arranged. If the flue gas contains 12 per cent $\mathrm{CO}_{2}$ and this bottle is lifted as the gas comes into it, so that the water levels in the jar and bottle are the same, the water will run down to the 88 cubic centimeters or per cent mark and the gas will be at atmospheric pressure as it has throughout the experiment. The other 12 per cent of the gas or the $\mathrm{CO}_{2}$ did not get through * Reproduced from Power.

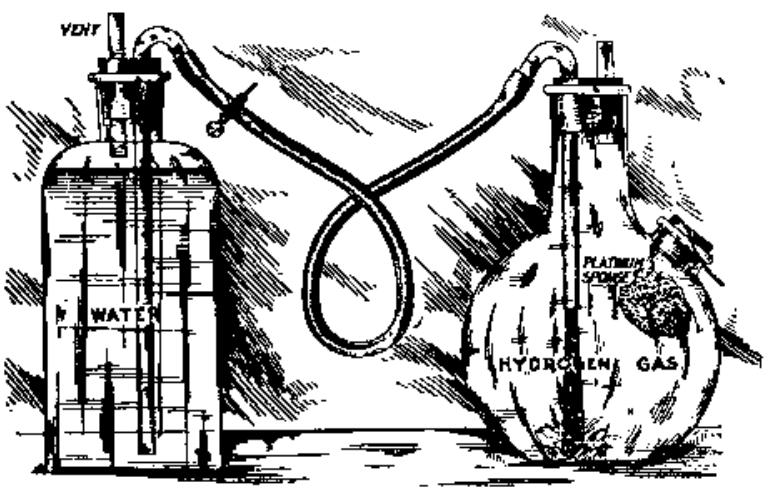

Fig. 1.-Absorption of $\mathrm{gas}$ by platinum sponge creates vacuum in bottle. a caustic-potash solution absorbs only the $\mathrm{CO}_{2}$ in the flue gases but allows the other gases to pass through. About the same process as that gone through in this last experiment goes on in a $\mathrm{CO}_{2}$ recorder.

In the recorder the chemical or "sponge" which absorbs the $\mathrm{CO}_{2}$ is caustic potash. The pen which marks the chart is attached to the top of a gas bell that rises and alls as it is filled with and emptied of the $\mathrm{CO}_{2}$ gas, just as the tanks at the gas works rise and fall.

Before continuing, let us understand why we say such and such a percentage of $\mathrm{CO}_{2}$. Percentage means "by the hundred," and 10 per cent or 12 per cent means 10 or 12 parts of a total of 100 parts. The total of 100 parts, or 100 per cent, may be any quantity we choose to make it. In measuring $\mathrm{CO}_{2}$, all or 100 per cent of the sample of gas taken from the flue is 100 cubic centimeters or a little over 6 cubic inches. When the flue-gas analysis shows 12 per cent $\mathrm{CO}_{2}$ we know that 12 parts, or 12 cubic shows 12 per cent $\mathrm{CO}_{2}$ we know that 12 parts, or 12 cubic
centimeters of the 100 parts, or 100 cubic centimeters, is $\mathrm{CO}_{2}$ gas

Fig. 3 shows the essentials of a $\mathrm{CO}_{2}$ recorder, and when you understand it, you will understand how and why any $\mathrm{CO}_{2}$ recorder works. The gas is brought from the boiler uptake and passed up through the filter, which contains a bottle nearly filled with oil or water to take out the soot in the sample. Gas flows into the measuring bottle, which holds exactly 100 cubic centimeters, or about 6 cubic inches. The sample having been measured, it goes to the "sponge," or caustic-potash solution, where all the $\mathrm{CO}_{2}$ gas is absorbed, and the rest bubbles through the solution and fills the receiver bottle. From the receiver bottle the gas is led to the gas bell, which has a water seal so that gas cannot escape to the atmosphere. This bell and the pen arm rise and mark the chart against which the pen bears. When the mark is made the gas is discharged to the atmosphere. The chart drum is revolved by clockwork, as usual.

As the caustic potash absorbs only $\mathrm{CO}_{2}$, we see that if the measured sample is high in $\mathrm{CO}_{2}$, there will be less gas going through the solution to fill the gas bell, and the pen will not make as long a mark as when more gas is passing through the solution. On a recording steam gage or thermometer the pen makes a longer mark as the presure or temperature increases from the zero point on the chart. This is not so with the $\mathrm{CO}_{2}$ recorder of this sort. The greater the $\mathrm{CO}_{2}$ percentage, the shorter the mark

To get the gas into and out of the measuring and reciving bottles, caustic-potash tanks, gas bell, etc., many siphons and traps are required. The motive power for most $\mathrm{CO}_{3}$ recorders is a stream of water flowing from a pet-cock. As the stream is increased the machine works faster; that is, it makes a greater number of readings per hour. With the usual $\mathrm{CO}_{2}$ machine about seven or eight readings per hour will produce a good chart.

\section{Organic Synthesis and Emil Fischer}

Since Wöhler's first synthesis of a natural organic compound, the chemist has succeeded in building up nearly all the natural compounds from their constituent elements in his laboratory; indeed, the synthesis of the sugars, the polypeptides, the alkaloids, uric acid and its derivatives are some of the greatest triumphs of the chemist. Much of the success in this field is due to the genius of Emil Fischer, and though he has cele brated his sixtieth birthday he shows no signs of relaxing his labors, being now responsible for another great chievement. The importance of the nucleus in the cell needs no emphasis, and therefore the value of the recent work, more particularly of Levene and his colaborators, in America, on its chemical composition has been widely recognized. In brief, the nucleic acids are composed of glucosidic compounds of purine derivatives combined with the carbohydrates to which phosphoric acid is also coupled. The synthesis of such a glucosidic compound of sugar and purine has long been essayed, but it is only now brought to a successful conclusion. Once the principle of the method of making them has been made clear all kinds of purine derivatives can be coupled with the carbohydrates, and when phosphoric acid has been introduced into the molecule the complete ynthesis of the nucleic acids will have been achieved.Nature.

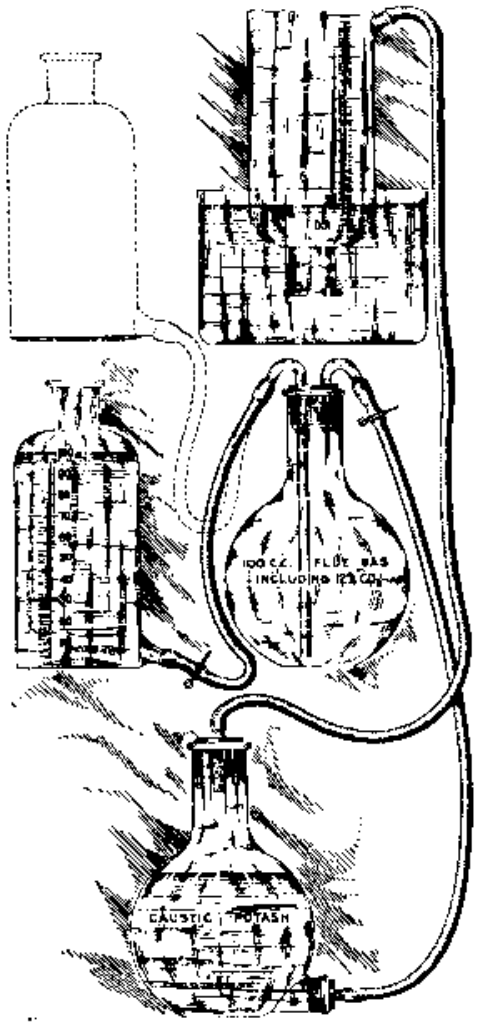

Fig. 2.-The caustic potash absorbs the $\mathrm{CO}_{2}$ but the other gases pass on.

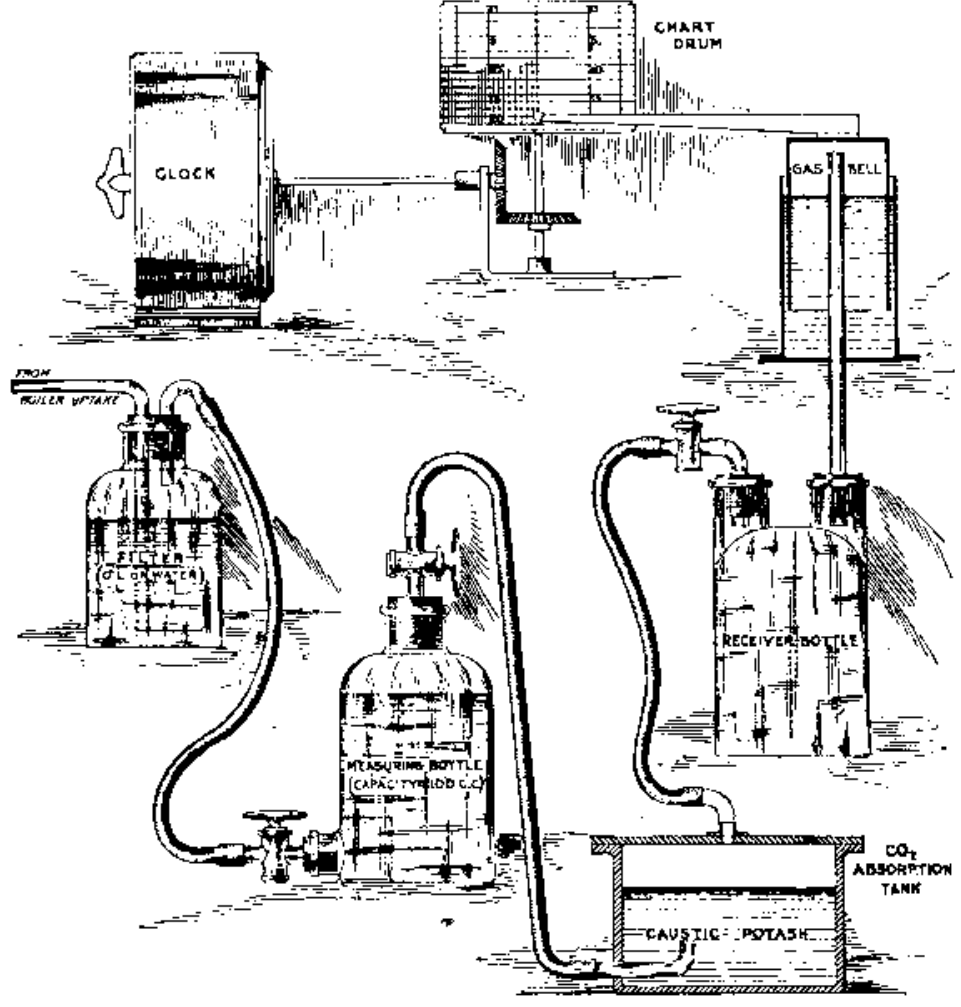

Fig. 3.-Here, in simple form, are the essentials of a carbon dioxide recorder. 